\title{
Multi-scale morphological descriptors from the fractal analysis of particle contour
}

by Giulia Guida*, Giulia M.B. Viggiani ${ }^{\dagger} \&$ Francesca Casini ${ }^{\ddagger}$

* PhD Student, Università degli Studi Niccolò Cusano, Italy, ORCID: 0000-0003-1129-7906

† Professor, Università degli Studi di Roma "Tor Vergata", Italy, ORCID: 0000-0002-0993-0322

* Professor, Università degli Studi di Roma “Tor Vergata”, Italy, ORCID: 0000-0001-7933-9055

\section{Contact Author:}

Giulia Guida

Università degli Studi Niccolò Cusano

Via Don Carlo Gnocchi 3

00166 Roma, ITALY

ph.: +39 3339389235, fax: +390645678379

e-mail: giulia.guida@unicusano.it

6170 words

2 tables

11 figures

\section{Keywords:}

Granular material, Shape, Fractals, Particle-scale, 


\begin{abstract}
The increasing understanding of the connection between particle morphology and mechanical behaviour of granular materials has generated significant research on the quantitative characterisation of particle shape. This work proposes a simple and effective method, based on the fractal analysis of their contour, to characterise the morphology of soil particles over the range of experimentally accessible scales. In this paper, three new non-dimensional quantitative morphological descriptors are introduced to describe (i) overall particle shape at the macro-scale, (ii) particle regularity at the meso-scale, and (iii) particle texture at the micro-scale. The characteristic size separating structural features and textural features emerges directly from the results of the fractal analysis of the contour of the particle, and is a decreasing fraction of particle dimension. To explore the meaning of the descriptors, the method is applied first to a variety of Euclidean smooth and artificially roughened regular shapes and then to four natural and artificial sands with different levels of irregularity. Relationships are established between the new morphological descriptors and other quantities commonly adopted in the technical literature.
\end{abstract}




\section{Introduction}

2 Besides relative density and effective stress, the mechanical behaviour of granular materials depends

3 on properties of both the aggregate and of constituent particles, such as particle size distribution,

4 mineral composition, inter-particle friction, hardness, strength, shape, and angularity. Experimental

5 data indicate that particle irregularity and surface roughness promote looser packing, affect small

6 strain stiffness, peak and critical state friction angles, compressibility, and creep behaviour (Youd,

7 1973; Miura et al., 1997; Santamarina \& Cascante, 1998; Cho et al., 2006; Bareither et al., 2008;

8 Chapuis, 2012; Cabalar et al., 2013; Kandasami \& Murthy, 2014; Altuhafi et al., 2016). Herle \&

9 Gudehus (1998) have proposed relationships between constitutive parameters and properties of grain

10 assemblies. Quite recently, Park and Santamarina (2017) argued that, as particle shape affects the

11 packing density of coarse-grained soils, it should be included in any meaningful soil classification

12 system.

13 Barret (1980) proposed that the shape of a particle may be described by three potentially independent 14 properties, namely overall form, angularity, and roughness, each referring to a characteristic scale.

15 Overall form carries information on the proportions of the particle at the macro-scale, i.e., on how 16 isometric or elongated the particle is; angularity accounts for local features of the particle at the meso-

17 scale; roughness describes the texture of the particle surface at the micro-scale (Cavarretta, 2009;

18 Mitchell \& Soga 2005; ISO, 2008).

19 Traditionally, in soil mechanics, particle shape is characterised as "angular" or "rounded" following

20 Powers (1953), or using reference charts such as that proposed by Krumbein and Sloss (1963), in

21 which paradigmatic shapes are arranged in a matrix whose rows and columns correspond to two 22 independent descriptors of sphericity (Krumbein, 1941) and roundness (Wadell, 1932). These can be 23 quantified by visual comparison of a given particle with the shapes in the matrix, but they are affected 24 by an element of subjectivity and provide at best an indication of particle morphology at the macroand meso-scale. 
Ehrlich and Weinberg (1970) and Meloy (1977) were among the first to use harmonic analysis of the 2D silhouette of a particle to obtain quantitative information on its shape. More recently, Mollon \& Zhao (2012; 2013) and Zhou et al. (2015) applied spherical harmonic analysis to characterise and reconstruct their morphology in 3D. It has been proposed that higher order harmonics may monitor grain roughness and surface texture, while lower order harmonics deal with overall particle shape (Bowman et al., 2001; Zhou et al., 2015). Meloy (1977) found that a linear relationship exists between the logarithm of the order of high frequency Fourier coefficients and the logarithm of their amplitude, somehow indicating a self-similar nature of texture.

It has been suggested that natural surfaces may have a multi-scale nature (Bhushan, 2001), selfsimilar over a broad range of scales (Richardson, 1961), so that a scale independent parameter, namely the fractal dimension, should carry a signature of the morphology of the outline of the particle. Arasan et al. (2011) proposed empirical relationships linking the fractal dimension of a particle's outline to more conventional morphology descriptors. The results by Orford and Whalley (1983), however, indicate that two or possibly more fractal elements emerge from the fractal analysis of the contour of natural grains, reflecting the morphological difference between micro-scale, or textural features, and meso-scale, or structural features.

The aim of this work is to propose a simple and effective method, based on the robust mathematical framework of fractal analysis, to characterise the morphology of soil particles in terms of three new quantitative descriptors that can be associated systematically to the observed mechanical behaviour of aggregates. and, if possible, bounded between zero and one, corresponding to the two extreme limits of noncompact shapes and extremely compact shapes, such as a circle. Several descriptors of overall form 
have been used in the literature (see e.g., Clayton et al. 2009 for a thorough review). Among others,

53 two-dimensional form descriptors include: e.g., bounding box ratio $B B R$, or the ratio between the minimum and maximum side of the edge tangent enclosing box; 2D sphericity $S$, or the ratio between the diameter of the maximum inscribed circle and the diameter of the minimum circumscribed circle; and circularity, or the ratio of the area of the shape to the area of a circle having the same perimeter, $C=4 \pi A / p^{2}$. While all these descriptors meet the requirements outlined above, only circularity is a true measure of the compactness of a 2D closed shape, while there are cases in which $B B R$ and $S$ may depend overly on one or two extreme points, or be unaffected by the presence of recesses. Different quantitative definitions of angularity, describing the local features of the particles boundary at the meso-scale, have been proposed (e.g. Wentworth, 1922; Wadell, 1932; Kuenen, 1956; Lees, 1964; Dobkinks \& Folk, 1970; Swan, 1974; Stachowiak, 1998). However, they all suffer from ambiguities related to the scale at which angularity should be computed. Along an irregular outline, in fact, the number of recognisable local features increases as the image magnification increases and it is not obvious how to distinguish between structural and textural local features. Wadell (1932) identified a "corner" as any portion of the projected outline of a particle which has a radius of curvature, $r$, less than or equal to the radius of the maximum inscribed circle, $R_{\max }$,in, and defined its roundness as the ratio of the two. He defined the overall degree of roundness of a particle as the arithmetic mean of the roundness of individual corners:

$70 \quad R=\frac{\sum r_{\mathrm{i}}}{N R_{\text {max }, \text { in }}}$

71 where $N$ is the total number of corners in the particle's outline. He recognised the problem of the 72 dependence of the computed value of roundness on the scale of observation, and suggested that 73 roundness should be computed on images of a standard size, somehow supporting the idea that the 74 characteristic scale of local features should be a proportion of the size of the particle. Zheng \& Hryciw (2015) used locally weighted regression and K-fold cross-validation to eliminate the effect of surface 
$81 \rho=\frac{S+R}{2}$ geometry.

$$
\rho=\frac{S+R}{2}
$$

Their experimental results and those from a very large database of published studies on several natural

roughness in the assessment of particle roundness by numerical methods based on computational

Cho et al. (2006) proposed to average the values of roundness and 2D sphericity, to obtain another morphological descriptor containing combined information on the macro and meso scales, which they called regularity:

and artificial sands indicate that several mechanical properties such as compressibility, void ratio extent, and small strain stiffness correlate well with particle regularity.

Finally, despite the increasing understanding of the role of roughness on the mechanical behaviour of coarse grained soils (e.g., Santamarina \& Cascante, 1998; Otsubo et al., 2016), very few well established procedures exist to characterise the higher order of irregularities and the characteristic scales to which they are associated. The traditional and most direct way to quantify roughness is in terms of the root mean square deviation of a surface or a profile from its average level. This requires high-resolution measurements of the surface, typically obtained by optical interferometry. Moreover, as sand particles are curved, unlike flat-engineered surfaces, the processing procedure must flatten the surface or the contour of the particle, in order to remove the influence of curvature on the computed values of roughness. This may be achieved by some motif extraction method, filtering regular features (such as waviness) from textural features (Boulanger, 1992; Yang et al., 2017) or by discretisation of the surface using best-fit planes of small size (Cavarretta et al., 2016). In both cases, however, the results depend on the shape motif parameters or the size of the best-fit planes.

The meaningful characteristic scale associated to textural features may depend on the mechanical parameter under examination; the works by Santamarina \& Cascante, (1998) and Otsubo et al. (2016) indicate that textural features at the micron-scale length may dominate the behaviour of contacts in 
101 by Yang \& Baudet (2016) and Yang et al. (2017) may be too minute to have an effect on the small 102 strain stiffness even at relatively low confining stress.

103 As discussed above, many authors have linked the definition of roughness to the fractal dimension of 104 either the outline of the particle (Anasar et al., 2011;Cavarretta, 2009; Hanaor et al., 2013) or in three105 dimensional analyses, of its surface (Yang and Baudet, 2016; Yang et al., 2017). The value of the 106 fractal dimension is theoretically bound between 1 and 2 in the first case and between 2 and 3 in the 107 second (Mandelbrot, 1975), where the lower bounds of the quoted ranges describe a perfectly smooth 108 shape, while the upper bounds correspond to extremely rough shapes.

\section{Fractal analysis}

\section{$111 \quad 3.1 \quad$ Method}

112 Fractal analysis stems from the observation that the measured length of the contour of many natural 113 irregular closed shapes, $p$, is a function of the measurement scale, $b$ (Mandelbrot, 1967), and that the 114 smaller the measurement scale, the longer the measured length becomes. The approximations with 115 segments of length $b$ of strictly self-similar mathematical curves, such as e.g., the Koch snowflake 116 (von Koch, 1904), have lengths:

$117 p=b^{(1-\alpha)}$

118 where $\alpha$ is the Hausdorff dimension, taking values between 1 and 2. Eq. (3) implies that the length 119 of the contour of any truly-fractal closed shape diverges to infinity as the measurement scale tends to 120 zero. When dealing with physical objects, indefinite subdivision of space does not make sense, as the 121 minimum measurement scale would be limited at least by the distance at the atomic level, while in 122 practice, well before this is achieved, it is limited by the experimental resolution with which the 123 contour of the particle is defined. However, the plot of the length of the contour of a particle, $p$, versus 124 measurement scale, $b$, still carries a signature of the morphology of the particle over the range of 125 experimentally accessible scales. At the upper end of this range, the characteristic dimension of the 
particle can be conventionally defined as the diameter of the circle having the same area as the 127 particle:

$$
D=\sqrt{\frac{4 A}{\pi}}
$$

129 The input for the fractal analysis is a 2D image of the particle of any resolution, as obtained, e.g., by 130 optical or scanning electron microscopy; it is evident that the higher the resolution of the image, the 131 more information can be extracted from the analysis. Typically, for natural silica sand, textural 132 features start to emerge at about $1 / 20$ of the characteristic size of the particle, so that, in order to be 133 able to observe them, it should be $b_{\min } / D<0.05$, where $b_{\min }$ is the minimum accessible scale of an 134 image of size $L$, and a number of pixels $N \times N$, and it is defined as the ratio between $L$ and $N$.

135 In order to obtain quantitative information from images, they were processed by contrast 136 enhancement, binarization and segmentation. Contrast enhancement increases image sharpness thus 137 facilitating subsequent binarization and segmentation. The method by Otsu (1979) was used to obtain 138 the binary version of the original greyscale image, by converting each pixel to either white 139 (foreground) or black (background), based on a threshold value. When the particles were not in 140 contact in the binarized image, they were identified simply by labelling areas composed by all 141 connected foreground pixels. In more complex situations, for instance when processing images 142 containing grains in contact, a watershed algorithm (Beucher, 1992) was used 143 for segmentation purposes. Figure 1a shows schematically a binarized particle image after 144 segmentation, in which white pixels correspond to the particle while black pixels correspond to the 145 background. The contour of the particle can be extracted by subtracting from the binarized image of 146 the particle its $8^{\text {th }}$-connected eroded, to obtain the external line of $8^{\text {th }}$-connected pixels (Fig. $1 \mathrm{~b}$ ) in the 147 form of a vector containing their coordinates $\left(x_{\mathrm{i}}, y_{\mathrm{i}}\right)$.

148 The algorithm, coded in Matlab (2015), computes the length of the contour adopting segments of 149 fixed length (Figure 1c-d). A point of the contour is chosen as the starting point for the calculation 150 (Fig. 1c) and one end of a segment of length $b$ is fixed to it. A simple "while" loop, which stops when 
151 the distance between the starting point and a successive point on the boundary is greater than or equal

152 to $b$, finds the intersection point between the other end of the segment and the boundary (Fig. 1c). In 153 turn, this intersection point becomes the starting point for the second segment, until the whole contour 154 is covered (Fig. 1d). Since a finite number of points discretize the outline, the exact distance between 155 two subsequent intersection points is not strictly equal to the segment length $b$, but the maximum 156 error is less than the pixel size. The loop ends when the distance between the intersection point and 157 the initial starting point is less than $b$ (Fig. 1e). The length of the contour is computed as the sum of 158 the distances between all the intersection points. As this length depends on the chosen initial starting 159 point (Stachowiak, 1998), consistently with the definition of the Hausdorff dimension, the procedure 160 is repeated using all the points of the boundary as starting points, and the perimeter of the particle, $p$, 161 is defined as the minimum computed value of the length of the contour. Finally, the normalised 162 perimeter, $p / D$, is plotted versus the corresponding normalised stick length, $b / D$, in a bi-logarithmic 163 plane.

\section{$164 \quad 3.2 \quad$ Three scales of information}

165 Figure 2 shows an example of the results of the fractal analysis applied to a natural grain of Toyoura sand. A SEM photograph of the sand grain at a magnification factor of $300 \times$ (Fig. 2a) was obtained

167 from Alshibli (2013). Figure 2b shows the same grain after binarization and segmentation; in this case, careful contrast enhancement was required to avoid altering the contour due to the presence of shadows and overlapping. The characteristic dimension of the particle is $D=185 \mu \mathrm{m}$ and the resolution of the image is 960 pixels $/ \mathrm{mm}$, or, in other words, one pixel corresponds to $b_{\min }=1.04 \mu \mathrm{m}$.

171 After boundary extraction, the perimeter was computed using segments of decreasing length from $b=D$ to $b=0.001 D$, see Figure $1 \mathrm{c}$.

173 Figure $2 \mathrm{~d}$ reports the results of the analysis in terms of $\log (b / D) v s \log (p / D)$. Starting from $b / D=1$ 174 and moving to the left, as $b / D$ decreases, $p / D$ increases rapidly and non-linearly from its minimum 175 value of 2 , corresponding to point (1) in Figure $2 \mathrm{~d}$. For smaller values of $b / D$, two linear trends can 
be identified, with slopes $-m$ and $-\mu$, until the computed perimeter saturates and the plot becomes horizontal when the segment length reaches the pixel size, $b_{\min } / D=0.0056$. The larger segment lengths, see e.g., points (1) to (3) in Figure 1d, even if providing the least accurate estimate of the actual perimeter of the particle, carry information about its overall proportions at the macro-scale. Intermediate segment lengths, see e.g., points (3) to (4), recognise the local features of the particle contour at the meso-scale, while small segment lengths, see e.g., point (5), convey the signature of surface texture, because they can follow the asperities of the contour at the micro-scale, see Figure 2c.

The results in Figure 2 are typical of natural sand particles. They confirm the findings by Orford and Whalley (1983) on the emergence of two distinct self-similar patterns describing structural and textural features. Moreover, the characteristic scale separating the two, which may be regarded as the maximum size of the micro-asperities, emerges directly from the results and corresponds to the stick length at the point of intersection of the two linear portions of the plot, $b_{\mathrm{m}}(=0.028 D=5.4 \mu \mathrm{m})$ in Figure 2d.

The absolute values of the slopes of the two linear portions of the plot relate to the fractal dimension in Eq. (3), and increase with the complexity of the profile (Vallejo, 1995). These can be obtained by automatic linear regression in the $\log (b / D): \log (p / D)$ plane, performing a check on the computed value of the coefficient of determination, $R^{2}$. Starting from $b_{\min } / D$, a linear regression is extended to include an increasing number of points, corresponding to larger and larger values of $b / D$, until $R^{2}$ remains approximately constant and equal to 1 . When $R^{2}$ decreases by more than $0.02 \%$, the process stops, and starts again with another regression on the remaining data; any linear trend must contain at least five data points. Figure 3 illustrates this procedure for the contour of the Toyoura sand particle of Figure 2. In this case, the first linear regression ends at a value of $b / D=b_{m} / D=0.028$ and the second linear regression at $b / D=0.30$, no further linear portions are identified by the algorithm. The computed absolute values of the slopes are $m=0.042\left(\alpha_{m}=1.042\right)$ and $\mu=0.137\left(\alpha_{\mu}=1.137\right)$. 
201 An important feature of the plot in Figure $2 \mathrm{~d}$ is the increase of the dimensionless perimeter above its 202 minimum value of 2 over the range of stick lengths connected to overall shape and structural features, $203\left(b_{\mathrm{m}} / D<b / D<1\right)$ :

$204 \Delta=(p / D)_{b_{m}}-2$

205 For the example in Figure 2, $\Delta=1.55$.

206 Starting from an input binarized image of the grain, with a resolution of $0.5-3 \mu \mathrm{m} / \mathrm{px}$, the Matlab 207 algorithm described in this section takes about $20 \mathrm{~s}$ on an ordinary pc to extract the boundary, run the 208 fractal analysis of the contour and recover the slopes of the fractal subsets.

\section{Simple shapes}

\section{$211 \quad 4.1 \quad$ Structural features: macro and meso scale}

212 Fractal analysis was applied first to the contour of simple smooth Euclidean shapes. Figure 4a shows 213 the results obtained for a set of shapes representing a smooth transition from a square to a circle, 214 obtained by rounding off the corners of the square with arcs of increasing radius, from 0.05 to 0.2 of 215 its side. The theoretical normalised perimeters of the circle and the square for an infinitesimal stick 216 length $(b / D \rightarrow 0)$ are $p / D=\pi(\approx 3.14)$ and $2 \times \pi^{0.5}(\approx 3.54)$ respectively.

217 The logarithm of the computed perimeter of the circle rises very rapidly with decreasing logarithm of 218 measurement length, reaching $98 \%$ of its asymptotic value at $b / D \approx 0.3$; for smaller measurement 219 lengths, the plot is linear and practically horizontal. This is because a smooth circle does not have any structural nor textural features and, therefore, the only linear portion of the plot is characterised by a

221 slope $m=\mu=0\left(\alpha_{m}=\alpha_{\mu}=1\right)$. On the other hand, the corners between the edges of the square act as 222 local features, giving rise to the emergence of a structural fractal subset at intermediate measurement 223 lengths $\left(m \neq 0, \alpha_{m}>1\right)$, which persists until the computed perimeter reaches about $97 \%$ of its 224 theoretical value. The plot then becomes nearly horizontal, $\left(\mu \approx 0, \alpha_{\mu} \approx 1\right)$, as, again, the figure is smooth. The slope of the "structural" subset is the same for all the rounded squares, $m=0.07$, while 
its extent reduces with increasing radius until it vanishes for the circle. This suggests that the fractal

227 dimension of the structural subset, $\alpha_{\mathrm{m}}(=1+m)$, may depend mainly on the angle existing between the

228 edges while its extent, $b_{\mathrm{m}}$, on the degree of roundness of the corners.

229 Figure $4 \mathrm{~b}$ reports the results obtained for a family of rectangles of increasing elongation. As far as 230 the rectangles are concerned, as expected, both the slope and the extent of the structural subset are 231 the same as those obtained for a square, $m=0.07$ and $b_{\mathrm{m}} / D=0.08$, because neither the degree of 232 acuteness, nor their roundness change with elongation. The computed value of the perimeter at $b_{\mathrm{m}} / D$, 233 always within $97 \%$ of the theoretical value, increases significantly with elongation.

\subsection{Textural features: microscale}

Figure 5 shows the results of the fractal analysis of three shapes obtained superimposing to a smooth circle of diameter $d$ an artificial saw-tooth profile consisting of equilateral triangles with decreasing side $l=\pi d / 50, \pi d / 100$, and $\pi d / 200$. The characteristic dimensions of the three shapes are $D=1.05 d$, $1.02 d$, and $1.01 d$, respectively. The computed normalised perimeter of the three shapes follows very closely that of the circle, until the measurement length reaches the dimension of the asperity; here the plot increases abruptly, attaining the theoretical value of the normalised perimeter, $p / D=5.97,6.12$, and 6.20, respectively. Despite some small oscillations, the fractal analysis is able to identify the size of the introduced asperities, although no fractal subset or linear portion of the plot emerges because the particle profile is not self-similar.

Figure $6 \mathrm{~g}$ shows the normalised perimeters of four Euclidean approximations of the Koch snowflake (Von Koch, 1906) at increasing order $n=2$ to 5 (see Figures 6a to f). The logarithm of the perimeter of each shape increases linearly with the logarithm of decreasing stick length and then it becomes constant at a value of $b / D_{\mathrm{n}}=L_{\mathrm{n}} / D_{\mathrm{n}}$, where $L_{\mathrm{n}}$ represents the length of the side of the order $n$ Euclidean approximation of the Koch snowflake. The slope of all plots is related to the fractal dimension of the

249 Koch snowflake, $-m_{\mathrm{koch}}=-0.262\left(\alpha_{\mathrm{Koch}}=1.262\right)$. Thus, the fractal analysis of the contour recognises both the complexity of the shape, which in this case is truly scale-independent, $(m=\mu)$ and the characteristic scale of the asperities, $L_{\mathrm{n}}$. 
252 The increase of the logarithm of the normalised perimeter is linear only if textural features are self253 similar; the fact that natural grains present one or more linear subsets indicates that their contour is 254 fractal over characteristic range of scales.

\section{Morphology descriptors}

Based on the results above, we propose that $\mu, m$, and $\Delta$, may be used to describe quantitatively the morphology of a particle at the micro-, meso-, and macro-scale, and that the characteristic length separating the textural and structural features may be taken as the value of $b_{\mathrm{m}}$, representing the maximum size of the micro-asperities.

Because the fractal dimension of a closed shape is theoretically bound between 1 and $2, \mu\left(=\alpha_{\mu}-1\right)$ and $m\left(=\alpha_{m}-1\right)$, are automatically bound between 0 and 1 , and can be effectively and directly used as morphology descriptors at the micro- and meso-scale. The increase of the dimensionless perimeter above its minimum value of 2 is theoretically the smallest for the circle:

$\Delta_{\text {circle }}=(p / D)_{b_{m}}-2 \approx \pi-2 \approx 1.14$

so that a morphology descriptor at the macro-scale, theoretically bound between 0 and 1 , can be defined as:

$M=\frac{\Delta_{\text {circle }}}{\Delta}$

269 To explore the meaning of these quantities and establish their relation with other descriptors adopted 270 in the literature, the fractal analysis was applied to the contour of the paradigmatic shapes included 271 in the chart by Krumbein and Sloss (1963) yielding the values of $m$ and $M$ reported in Figure 7. Each 272 particle in the chart has a dimension of about $10-15 \mathrm{~mm}$, and the resolution of the images is of the order of 3 pixels $/ \mathrm{mm}$, far too low to appreciate textural features.

Figure 8a shows that, both for simple shapes and smooth figures such as those in Krumbein and Sloss (1963) chart, descriptor $M$ is a very close measure of circularity, $C\left(=4 \pi A / p^{2}\right)$. For complex shapes, 
definition contains the perimeter of the particle, which increases with decreasing scale of observation.

278 However, because descriptor $M$ is evaluated over the range $b_{\mathrm{m}}<b<D$ it accounts only for macroand meso- features and is therefore effectively independent of the scale of observation.

Figure 8b shows that for the shapes in Krumbein and Sloss (1963) chart, descriptor $m$ correlates very well with $\rho$, and, therefore, it is likely to control all those aspects of the mechanical behaviour that depend on regularity, such as compressibility, void ratio extent, and small strain stiffness.

\section{Real particles}

285 Figure 9 shows nine SEM photographs of the grains of three natural sands with different morphological features (Alshibli, 2013).

From a preliminary morphology analysis, conducted using standard charts, ASTM Sand grains (Fig. 9a), can be classified as rounded and regular $(\rho \approx 0.9)$, Columbia Grout grains (Fig. 9b) as subangular and less regular $(\rho \approx 0.6)$ and finally Toyoura Sand grains (Fig. $9 \mathrm{c})$ as angular and even less $\operatorname{regular}(\rho \approx 0.5)$.

The value of $b_{\min } / D$ is about the same for all three sands. The image of ASTM Sand has a lower resolution (380 pixels $/ \mathrm{mm})$ than those of Columbia Grout and Toyoura sands (960 pixels $/ \mathrm{mm})$, but the grain size is larger for ASTM sand, $D \sim 850 \mu \mathrm{m}$, and smaller for the other two, ranging between 297 and $420 \mu \mathrm{m}$, and between $100 \mu \mathrm{m}$ and $320 \mu \mathrm{m}$, respectively.

Figure 9 and Table 1 summarise the results of the fractal analysis of the contour of the nine sand particles, obtained following the procedures outlines in Section 3. The results for the three ASTM Sand grains (Fig. 9a), at least for $b / D \geq 0.05$, overlap with one another and are very close to those obtained for a circle, with very small values of $m(\approx 0.02)$ denoting the absence of structural features. For grains (1) and (3) the almost horizontal linear portion of the plot extends down to $b_{\mathrm{m}} / D \approx 0.03$, 300 and then the plot starts to increase linearly $(\mu \approx 0.05)$ as the textural features begin to contribute to 301 the computed value of the normalised perimeter. The extent of the structural subset for grain (2) is 
more limited. In this case, the textural subset emerges at a value of $b_{\mathrm{m}} / D \approx 0.05$ with $\mu \approx 0.12$, almost twice the value computed for the other two grains, which is an index of a textural complexity not easily recognisable by naked eye from the images in Figure 9a. The three grains have very similar and high values of $M=0.84-0.95$, consistent with the isometry of their overall shape, with the highest value associated to grain (3), easily recognised as the most circular in Figure 9a.

The normalised perimeters of grains (2) and (3) of Columbia Grout (Fig. 9b) are very similar. Two clearly identifiable fractal subsets emerge from the fractal analysis of their contour, with $m \approx 0.04$ and $\mu \approx 0.11$, and the same cut-off separating structural and textural features, $b_{\mathrm{m}} / D=0.014$. For grain (1), only one fractal subset emerges with $\mu \approx 0.19$ and it is impossible to identify clearly the

311 characteristic scale separating structural from textural effects. In fact, closer inspection of the SEM 312 photographs in Figure $9 \mathrm{~b}$ reveals that the contour of grain (1) is characterised by asperities of a larger 313 maximum size than for the other two grains, so that the increase of normalised perimeter due to 314 texture complexity masks in part the effects of structural features. In cases like this, it is difficult to 315 define unambiguously two different linear trends: the descriptor $M$ is computed using a value of 316 normalised perimeter at $b_{\mathrm{m}} / D=0.1$ corresponding to the end of the linear regression. On average, 317 Columbia Grout grains are less circular than ASTM sand, with smaller values of $M(\approx 0.75)$. It must 318 be noted that, for grain (1) the portion of the contour corresponding to the shadowed area in the 319 greyscale image is very irregular, possibly due to inaccuracies in the automatic segmenting 320 procedures that did not recognize distinctly the grain contour. To a certain extent, the same comment 321 applies also to grain (2) of ASTM sand.

322 Two fractal subsets, characterised by $\mu \approx 0.10$ and $m \approx 0.05$, emerge from the results obtained for all 323 three Toyoura sand grains (Fig. 7c). However, the structural subset for grain (3) extends to smaller 324 scales $\left(b_{\mathrm{m}} / D \approx 0.03\right)$ than for grains (1) and (2) $\left(b_{\mathrm{m}} / D \approx 0.07\right.$ and 0.08 , respectively) denoting a 325 smaller maximum size of textural asperities $\left(b_{\mathrm{m}} \approx 6.7 \mu \mathrm{m}\right)$, which would have been difficult to detect 
by naked eye. Moreover, because grain (3) is more elongated than the other two, its normalised

327 perimeter increases more and has a smaller value of $M(\approx 0.6)$.

328 The fractal dimensions of the structural and textural subset of the three natural sands given above 329 compare favourably with those reported by Orford and Whalley (1983) for carbonate beach grains 330 from the Maldives and pyroclastic particles from the 1980 Eruption of Mount St Helens.

331 The sand grains in Figure 9 were chosen intentionally so that two of them would be representative of 332 the typical results for each material and one would deviate from typical in one or more respects. These 333 deviations may be due to an occasional difference in form, as in the case of the much more elongated 334 grain (3) of Toyoura Sand, or by an occasional increase of complexity of the contour, as in the case 335 of grain (2) of ASTM sand, be it true or due to errors in image processing due to the presence of 336 shadows, as partly for grain (1) of Columbia Grout. This is an indication of the power of the method, 337 which is very sensitive even to very small variations in the complexity of the contour.

338 Figure 10 shows nine SEM photographs of grains of a crushed Light Expanded Clay Aggregate 339 (LECA) (see e.g. Casini et al., 2013), in the sizes $500-1000 \mu \mathrm{m}$ or "large" (Fig. 10a), 125 - $250 \mu \mathrm{m}$ 340 or "intermediate" (Fig. 10b), and $<63 \mu \mathrm{m}$ or "fine" (Fig. 10c). The SEM images have a resolution of 341340,686 and 3430 pixels/mm, for large, intermediate, and small grains, respectively.

342 The angularity of crushed LECA particles (Figure 10) increases from sub-angular to very angular and 343 their regularity reduces from $\rho \approx 0.5$ to $\rho \approx 0.4$ with decreasing grain size; due to exposed intra344 granular porosity, the particles are visually very rough if compared to natural sand grains.

345 Fractal analysis permits to appreciate the different morphological characters of the three grain sizes, 346 see Table 2. For all three large grains (Fig. 10a), two fractal subsets emerge from the data, with similar 347 values of $m \approx 0.09-0.13$ and $\mu \approx 0.15-0.20$, and cut-off between structural and textural feature, $348 b_{\mathrm{m}} / D \approx 0.05-0.08$. The only significant difference between the three grains is their elongation, which 349 is minimum for grain (2), with the largest value of $M \approx 0.64$. On the other hand, both for intermediate 350 (Fig. 10b) and small (Fig. 10c) LECA, only one fractal subset emerges with $\mu \approx 0.12-0.15$ and it is 351 impossible to identify a characteristic scale separating structural from textural effects. Similarly to 
what happened for grain (1) of Columbia Grout, for both "small" and "intermediate" LECA, the

353 increase of normalised perimeter due to texture complexity overlaps with the increase of perimeter 354 due to structural features. It is interesting that, the slope of the textural subset is the same for all grain 355 sizes, see Table 2, indicating that the complexity of the contour of fine fragments is the same as that 356 of large grains and that the texture of crushed LECA is self-similar down to a scale of asperities of 357 the order of $0.3 \mu \mathrm{m}$. Consistently with what done before, $M$ was computed using the value of 358 normalised perimeter before textural features begin to affect significantly the results, i.e., at $359 \quad b_{\mathrm{m}} / D \approx 0.12-0.29$.

360 The high fractal dimension of the textural LECA subset are comparable to those obtained by Orford 361 and Whalley (1983) for highly irregular particles with crenellate morphology such as e.g., carbonate 362 cemented pure quartz sandstones or radiolaria of micro-granular quartz in a matrix of ferruginous 363 quartz.

364 Figure 11 shows that, for the natural and artificial sand grains considered in this study, the normalised maximum size of microstructural features, $b_{\mathrm{m}} / D$, decreases with increasing equivalent diameter, $D$.

366 For small particles $(D<100 \mu \mathrm{m})$, textural asperities have a characteristic size of between $15 \%$ and $36730 \%$ of the dimension of the particle and, hence, play simultaneously a structural and textural role.

\section{Discussion}

370 The method discussed above examines the contour of 2D images of particles. This is convenient, 371 because images from very different sources such as e.g., SE or optical microscope photographs of 372 particles or of thin sections, are easy to obtain. However, it is necessary to address some issues of 373 meaningfulness of results, resolution, and errors connected to image processing.

374 The main problem of working with $2 \mathrm{D}$ images is that particles tend to lie flat on their major 375 dimensions, introducing a bias in their orientation. This may be overcome by scanning particles 376 allowed to fall under gravity, at a controlled rate, between a laser and a high speed camera (Sympatec, 377 2008), so that an outline of the particle can be recorded at random orientation (Altuhafi et al., 2013). 
378 However, even in this case, differences have been reported between both the average values and the 379 distribution of computed 2D and 3D morphology descriptors (Fonseca et al., 2012). This is possibly 380 because, when working with 2D images, the outline is evaluated on the projection of the particle, and 381 thus multi-level asperities are flattened in one plane, altering the real particle profile.

382 In recent years, the development of technology and image processing methods has greatly increased 383 the ability to characterise the microstructure of granular materials in 3D (e.g. Lin \& Miller, 2005; Al384 Raoush, 2007; Bagheri et al., 2015; Devarreawaere et al., 2015; Yang et al., 2017; Zhou et al., 2018). 385 However, full 3D characterization of particle morphology requires sophisticated computational tools, 386 significant data storage resources, and advanced experimental techniques, such as electron 387 interferometry and X-ray tomography, which are not readily available in the common geotechnical 388 laboratories, with the result that, in practice, charts remain still the most commonly used method of 389 estimating particle shape.

390 It is evident that the proposed method can be applied to any $2 \mathrm{D}$ image, including polar sections of 391 three-dimensional reconstructions of particles obtained by X-ray tomography or advanced optical 392 microscopy, and that the range of experimentally accessible scales depends on the resolution of the 393 input image. SEM images have higher resolutions, of the order of about 1-3 $\mu \mathrm{m} / \mathrm{pixel}$, than images 394 obtained from other sources; basic micro 3D tomography typically reach resolutions of about $39510 \mu \mathrm{m} /$ pixel, while dynamically or statically acquired optical images only of about $20 \mu \mathrm{m} / \mathrm{pixel}$. 396 Information about surface texture can only be retrieved by high-resolution images, whereas, if meso397 and macro- scale information is required, it is possible to use also lower resolution imaging 398 techniques.

399 As highlighted by the examples discussed above, contrast enhancement, thresholding and 400 segmentation techniques all play a role in the quality of the obtained results. Although these 401 processes are automatic, they require calibration, which may be time consuming. This is less critical 402 for e.g., thin sections and dynamically acquired images that are usually well in contrast, but externally sourced SEM images and polar sections of 3D reconstructions of particles require careful 
calibration of the image processing procedures, because of noise, particles contacts, poor contrast, 405 shadows and overlapping. It is very difficult to give general recipes, and each case will have to be considered based on the specific needs.

\section{Conclusions}

409 Fractal analysis is a simple, quantitative, and effective method to describe particle shape over the 410 range of experimentally accessible scales. Its application to smooth and artificially roughened simple 411 shapes permitted to define three quantitative non-dimensional descriptors, $M$, $m$, and $\mu$, to 412 characterise particle morphology at the macro-, meso-, and micro-scale, respectively.

413 Descriptor $M$, or the normalised initial increase of the perimeter ratio $(p / D)$, is a very close measure 414 of circularity; as it accounts only for overall form and structural features, it is effectively independent 415 of the scale of observation. Descriptor $m$, or the fractal dimension of the structural subset, may be considered as an "irregularity" index at the meso-scale. Descriptor $\mu$, or the fractal dimension of the textural subset, increases with the complexity of the contour, and may be used as a texture index, 418 together with the maximum size of the micro-asperities, which emerges from the results of the fractal 419 analysis.

420 Application of the method to sand grains of different origin, size, angularity, and regularity, yielded 421 very convincing results in terms of its ability to identify their key morphological characters. For most 422 grains, the cut-off size of asperities separating textural from structural features emerges clearly from 423 the results as the characteristic length corresponding to the intersection point of the textural and 424 structural subsets. However, there are instances in which only one fractal subset emerges from the 425 data, either because the increase of normalised perimeter due to relatively large micro-asperities masks the structural subset, or because, for very small particles, the particle contour is indeed self427 similar over the entire range of accessible scales, as the maximum size of the asperities is comparable 428 to the equivalent dimension of the grain. 
429 Based on the idea that particle morphology is a signature of the formation processes, geologists have 430 used particle shape to identify the geological origin and discriminate between sedimentary 431 environments (e.g., Ehrlich \&Weinberg, 1970; Demirmen, 1972; Orford \& Whalley, 1983). From the 432 perspective of geotechnical engineering, it will be useful to associate these quantitative morphology 433 descriptors to different aspects of the observed mechanical behaviour, such as, e.g., compressibility, 434 stiffness and strength (e.g., Miura et al., 1997; Cho et al., 2006).

435 It is evident that, as the shape of individual grains of any natural sand is variable, their morphology 436 can only be characterised in terms of average values and standard deviations of the descriptors for 437 statistically representative grain populations.

438 The method we propose is relatively simple, has low computation effort, and can be applied to 2D 439 images of any resolution, from very low to very high. More information can be extracted as the 440 resolution of the images increases. It is possible that, as suggested by Orford and Whalley (1983), 441 using higher-resolution images more than two fractal subsets would emerge. 


\section{REFERENCES}

445 Al-Raoush, R. (2007). Microstructure characterization of granular materials. Physica A: Statistical mechanics and its Applications, 377(2): 545-558.

Alshibli, K. (2013). The University of Tennessy. Retrieved July 24, 2017, from http://web.utk.edu/ alshibli/research/MGM/archives.php.

Altuhafi, F., O'sullivan, C., \& Cavarretta, I. (2013). Analysis of an image-based method to quantify the size and shape of sand particles. Journal of Geotechnical and. Geoenvironmental Engineering, 139(8): 1290-1307.

Altuhafi, F.N., Coop, M.R., \& Georgiannou, V.N. (2016). Effect of Particle Shape on the Mechanical Behavior of Natural Sands. Journal of Geotechnical and. Geoenvironmental Engineering, 142(12): 04016071.

Arasan, S., Akbulut, S., \& Hasiloglu, A. S. (2011). The relationship between the fractal dimension and shape properties of particles. KSCE Journal of Civil Engineering, 15(7): 1219.

Bagheri, G. H., Bonadonna, C., Manzella, I., \& Vonlanthen, P. (2015). On the characterization of size and shape of irregular particles. Powder Technology, 270, 141-153.

Bareither, C. A., Edil, T. B., Benson, C. H. \& Mickelson, D. M. (2008). Geological and physical factors affecting the friction angle of compacted sands. Journal of Geotechnical and. Geoenvironmental Engineering, ASCE, 134(10): 1476-1489.

Barrett, P.J. (1980). The shape of rock particles, a critical review. Sedimentology, 27(3): 291-303.

Beucher, S., \& Meyer, F. (1992). The morphological approach to segmentation: the watershed transformation. Optical Engineering-New York-Marcel Dekker Incorporated, 34: 433-433.

Boulanger, J. (1992). The "Motifs" method: An interesting complement to ISO parameters for some functional problems. International Journal of Machine Tools and Manufacture, 32(1-2): 203-209. descriptors analysis. Géotechnique, 51(6): 545-554. 
Bhushan, B. (2001). Nano-to microscale wear and mechanical characterization using scanning probe microscopy. Wear, 251(1): 1105-1123.

Cabalar, A. F., Dulundu, K. \& Tuncay, K. (2013). Strength of various sands in triaxial and cyclic direct shear tests. Engineering Geolology, 156(1): 92-102.

Casini, F., Viggiani, G.M.B., \& Springman S.M. (2013). Breakage of an artificial crushable material under loading. Granular Matter, 15(5): 661-673.

Cavarretta, I. (2009). The influence of particle characteristics on the engineering behaviour of granular materials. PhD thesis, Imperial College, London.

Cavarretta, I., O'Sullivan, C., \& Coop, M. R. (2016). The relevance of roundness to the crushing strength of granular materials. Géotechnique, 67(4): 301-312.

Chapuis, R. P. (2012). Estimating the in situ porosity of sandy soils sampled in boreholes. Engineering Geology, 141-142(19): 57-64.

Cho, G.C., Dodds, J., \& Santamarina, J.C. (2006). Particle shape effects on packing density, stiffness, and strength: natural and crushed sands. Journal of Geotechnical and. Geoenvironmental Engineering , ASCE, 132(5): 591-602.

Clark, M.W. (1981). Quantitative shape analysis: a review. Mathematical Geology, 13(4): 303-320.

Clayton, C. R. I., Abbireddy, C. O. R. \& Schiebel, R. (2009). A method of estimating the form of coarse particulates. Géotechnique, 59(6): 493-501.

Demirmen, F. (1972). Mathematical search procedures in facies modelling in sedimentary rocks. In: Mathematical Modelling of Sedimentary Processes. Plenum Press, New York, 81-114.

Devarrewaere, W., Foqué, D., Heimbach, U., Cantre, D., Nicolai, B., Nuyttens, D., \& Verboven, P. (2015). Quantitative 3D shape description of dust particles from treated seeds by means of X-ray micro-CT. Environmental science \& technology, 49(12), 7310-7318.

DobkinsJr, J.E., \& Folk, R.L. (1970). Shape development on Tahiti-nui. Journal of Sedimentary Research, 40(4). 
Ehrlich, R., \& Weinberg, B. (1970). An exact method for characterization of grain shape. Journal of Sedimentary Research, 40(1).

496 Fonseca, J., O’Sullivan, C., Coop,M. \& Lee, P. (2012). Non-invasive characterization of particle morphology of natural sands. Soils Foundation, 52(4): 712-722.

Hanaor, D.A., Gan, Y., \& Einav, I. (2013). Effects of surface structure deformation on static friction at fractal interfaces. Géotechnique Letters, 3(2): 52-58.

Herle I, Gudehus G (1999) Determination of parameters of a hypoplastic constitutive model from

ISO (2008). ISO 9276-6:2008: Representation of results of particle size analysis - Part 6: descriptive and qualitative representation of particle shape and morphology. Geneva, Switzerland: ISO.

Kandasami, R. K. \& Murthy, T. G. (2014). Effect of particle shape on the mechanical response of a granular ensemble. In Geomechanics from micro to macro (eds K. Soga, K. Kumar, G. Biscontin and M. Kuo), London, UK: CRC Press: 1093-1098.

Krumbein, W.C. (1941). Measurement and geological significance of shape and roundness of sedimentary particles. Journal of Sedimentary Research, 11(2): 64-72.

509 Krumbein, W.C., \& Sloss, L.L. (1963). Stratigraphy and sedimentation. $2^{\text {nd }}$ ed. WH Freeman \& Co., $510 \quad$ San Francisco, California, USA.

511 Kuenen, P.H. (1956). Experimental abrasion of pebbles: 2. Rolling by current. Journal of Geology, $512 \quad$ 64(4): $336-368$.

513 Lees, G. (1964). A new method for determining the angularity of particles. Sedimentology, 3(1): 251421.

515 Lin, C. L., \& Miller, J. D. (2005). 3D characterization and analysis of particle shape using X-ray microtomography (XMT). Powder Technology, 154(1), 61-69.

517 Mandelbrot, B. B. (1967). How long is the coast of Britain. Science, 156(3775): 636-638. 
518 Mandelbrot, B. B. (1975). Stochastic models for the Earth's relief, the shape and the fractal dimension 519 of the coastlines, and the number-area rule for islands. Proceedings of the National Academy of $520 \quad$ Sciences, 72(10): 3825-3828.

521 Matlab 2015b (2015). [Computer Software] Matworks.

522 Meloy, T. P. (1977). Fast Fourier transforms applied to shape analysis of particle silhouettes to obtain 523 morphological data. Powder Technology, 17(1): 27-35.

524 Miura, K., Maeda, K., Furukawa, M., \& Toki, S. (1997). Physical characteristics of sands with different primary properties. Soils Foundation, 37(3): 53-64.

Mitchell, J.K., Soga, K. (2005). Fundamentals of soil behaviour. $3^{\text {rd }}$ edition, New York, USA:Wiley.

Mollon, G., \& Zhao, J. (2012). Fourier-Voronoi-based generation of realistic samples for discrete modelling of granular materials. Granular matter, 14(5), 621-638.

Mollon, G., \& Zhao, J. (2013). Generating realistic 3D sand particles using Fourier descriptors. Granular Matter, 15(1), 95-108.

Orford, J.D., \& Whalley, W.B. (1983). The use of the fractal dimension to quantify the morphology of irregular-shaped particles. Sedimentology, 30(5): 655-668.

Otsu, N. (1979). A threshold selection method from gray-level histograms. IEEE transactions on systems, man, and cybernetics, 9(1): 62-66.

Otsubo, M., O'Sullivan, C., Hanley, K.J., \& Sim, W.W. (2016). The influence of particle surface roughness on elastic stiffness and dynamic response. Géotechnique, 67(5): 452-459.

Park, J., \& Santamarina, J. C. (2017). Revised soil classification system for coarse-fine mixtures. Journal of Geotechnical and Geoenvironmental Engineering, 143(8): 04017039.

539 Powers, M.C. (1953). A new roundness scale for sedimentary particles. J. Sedimentary Research, 23(2): 117-119.

541 Richardson, L.F. (1961). The problem of contiguity. General systems yearbook, 6: 139-187.

542 Santamarina, J.C., \& Cascante, G. (1998). Effect of surface roughness on wave propagation parameters. Géotechnique, 48(1): 129-136. 
544 Sympatec. (2008). QICPIC. Windox-operating instructions release 5.4.1.0. Sympatec, Clausthal545 Zellerfeld, Germany.

546 Stachowiak, G.W. (1998). Numerical characterization of wear particles morphology and angularity 547 of particles and surfaces. Tribology International, 31(1): 139-157.

548 Swan, B. (1974). Measures of particle roundness: a note. Journal of Sedimentary Research, 44(2): $549 \quad 572-577$.

550 Vallejo, L.E. (1995). Fractal analysis of granular materials. Géotechnique, 45(1): 159-163.

551 Von Koch, H. (1906). Sur une courbe continue sans tangente, obtenue par une construction 552 géométrique élémentaire pour l'étude de certaines questions de la théorie des courbes planes. Acta $553 \quad$ Mathematica, 30:145-174.

554 Wadell, H. (1932). Volume, shape, and roundness of rock particles. Journal of Geology, 40(5): 443$555 \quad 451$.

556 Wentworth, C.K. (1922). A scale of grade and class terms for clastic sediments. Journal of Geology, $557 \quad 30(5): 377-392$.

558 Yang, H., \& Baudet, B.A. (2016). Characterisation of the Roughness of Sand Particles. Procedia $559 \quad$ Engineering, 158: 98-103.

560 Yang H., Baudet B.A., \& Yao T. (2017). Characterization of the surface roughness of sand particles 561 using an advanced fractal approach. Proceeding of Royale Society of London A, 472: 20160524.

562 Youd, T.L. (1973). Factors controlling maximum and minimum densities of sands. In: Evaluation of 563 relative density and its role in geotechnical projects involving cohesionless soils, ASTM STP, $564 \quad$ 523: $98-112$.

565 Zheng, J. \& Hryciw, R. (2015). Traditional soil particle sphericity, roundness and surface roughness 566 by computational geometry. Géotechnique, 65(6): 494-506.

567 Zhou, B., Wang J (2015). Random generation of natural sand assembly using micro X-ray 568 tomography and spherical harmonics. Géotechnique Letters, 5(1): 6-11. 
569 Zhou, B., Wang J, Wang H (2018). Three-dimensional sphericity, roundness and fractal dimension $570 \quad$ of sand particles. Géotechnique, 68(1): 18-30. 
Table 1. Results of fractal analysis of natural sand particles

\begin{tabular}{|c|c|c|c|c|c|c|c|c|c|}
\hline Material & $\#$ & $\begin{array}{c}D \\
{[\mu \mathrm{m}]}\end{array}$ & $\begin{array}{c}b_{\min } / D \\
{[-]}\end{array}$ & $\begin{array}{c}b_{\min } \\
{[\mu \mathrm{m}]}\end{array}$ & $\begin{array}{c}b_{\mathrm{m}} / D \\
{[-]}\end{array}$ & $\begin{array}{c}b_{\mathrm{m}} \\
{[\mu \mathrm{m}]}\end{array}$ & $\begin{array}{c}\mu \\
{[-]}\end{array}$ & $\begin{array}{l}m \\
{[-]}\end{array}$ & $\begin{array}{l}M \\
{[-]}\end{array}$ \\
\hline \multirow{3}{*}{ ASTM Sand } & 1 & 757.1 & 0.003 & 2.6 & 0.024 & 18.3 & 0.04 & 0.01 & 0.87 \\
\hline & 2 & 929.5 & 0.002 & 2.2 & 0.049 & 45.7 & 0.12 & 0.03 & 0.84 \\
\hline & 3 & 921.3 & 0.002 & 2.2 & 0.034 & 31.8 & 0.06 & 0.02 & 0.95 \\
\hline \multirow{3}{*}{$\begin{array}{l}\text { Columbia } \\
\text { Grout }\end{array}$} & 1 & 333.2 & 0.003 & 1.0 & 0.142 & 47.4 & 0.19 & - & 0.83 \\
\hline & 2 & 401.4 & 0.002 & 1.0 & 0.017 & 6.8 & 0.11 & 0.03 & 0.79 \\
\hline & 3 & 395.7 & 0.002 & 1.0 & 0.017 & 6.7 & 0.11 & 0.05 & 0.66 \\
\hline \multirow{3}{*}{ Toyoura Sand } & 1 & 223.9 & 0.004 & 0.9 & 0.070 & 15.6 & 0.11 & 0.05 & 0.76 \\
\hline & 2 & 225.8 & 0.004 & 0.9 & 0.083 & 18.8 & 0.09 & 0.05 & 0.81 \\
\hline & 3 & 315.4 & 0.003 & 0.9 & 0.029 & 9.1 & 0.08 & 0.05 & 0.59 \\
\hline
\end{tabular}


Table 2. Results of fractal analysis of crushed LECA particles of decreasing size

\begin{tabular}{|c|c|c|c|c|c|c|c|c|c|}
\hline Material & $\#$ & $\begin{array}{c}D \\
{[\mu \mathrm{m}]}\end{array}$ & $\begin{array}{c}b_{\min } / D \\
{[-]}\end{array}$ & $\begin{array}{c}b_{\min } \\
{[\mu \mathrm{m}]}\end{array}$ & $\begin{array}{c}b_{\mathrm{m}} / D \\
{[-]}\end{array}$ & $\begin{array}{c}b_{\mathrm{m}} \\
{[\mu \mathrm{m}]}\end{array}$ & $\begin{array}{c}\mu \\
{[-]}\end{array}$ & $\begin{array}{l}m \\
{[-]}\end{array}$ & $\begin{array}{l}M \\
{[-]}\end{array}$ \\
\hline \multirow{3}{*}{$\begin{array}{c}\text { LECA } \\
500-1000 \mu \mathrm{m}\end{array}$} & 1 & 759.9 & 0.003 & 2.6 & 0.049 & 37.3 & 0.20 & 0.09 & 0.53 \\
\hline & 2 & 742.2 & 0.003 & 2.6 & 0.049 & 36.4 & 0.15 & 0.09 & 0.64 \\
\hline & 3 & 636.9 & 0.004 & 2.6 & 0.083 & 53.0 & 0.15 & 0.13 & 0.54 \\
\hline \multirow{3}{*}{$\begin{array}{c}\text { LECA } \\
125-250 \mu \mathrm{m}\end{array}$} & 1 & 190.2 & 0.007 & 1.3 & 0.141 & 26.9 & 0.14 & - & 0.66 \\
\hline & 2 & 191.0 & 0.007 & 1.3 & 0.169 & 32.2 & 0.13 & - & 0.63 \\
\hline & 3 & 155.5 & 0.008 & 1.3 & 0.119 & 18.5 & 0.15 & - & 0.73 \\
\hline \multirow{3}{*}{$\begin{array}{l}\text { LECA } \\
<63 \mu \mathrm{m}\end{array}$} & 1 & 26.2 & 0.010 & 0.3 & 0.168 & 4.4 & 0.13 & - & 0.61 \\
\hline & 2 & 20.6 & 0.012 & 0.2 & 0.169 & 3.5 & 0.12 & - & 0.71 \\
\hline & 3 & 26.4 & 0.010 & 0.3 & 0.287 & 7.6 & 0.12 & - & 0.74 \\
\hline
\end{tabular}



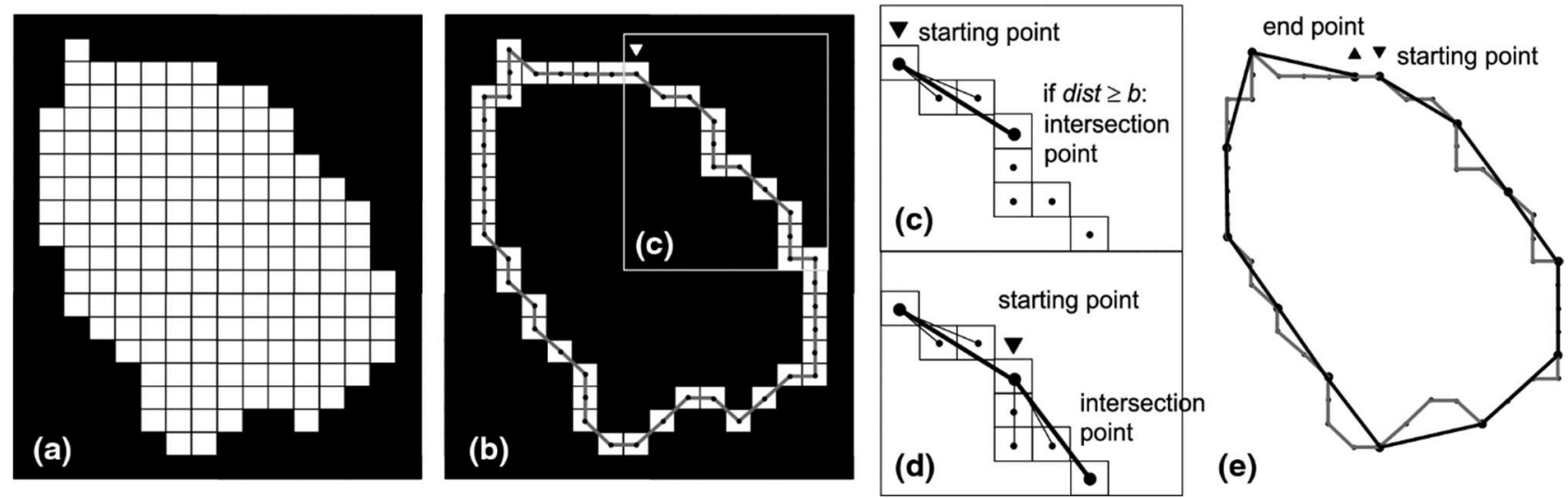

Figure 1. Steps of the method: (a) thresholding and segmentation of image, (b) extaction of 8thconnected boundary, (c), (d) and (e) measurement of perimeter with segments of fixed length.

(a)

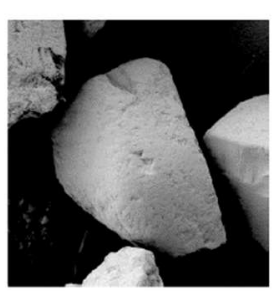

(b)

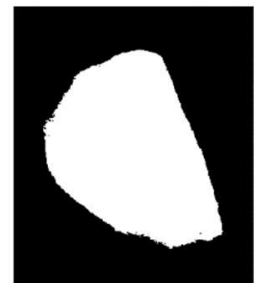

(1) $b / D=1.000$

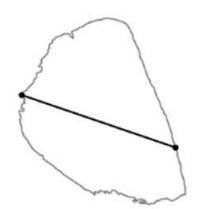

(4) $b / D=0.050$

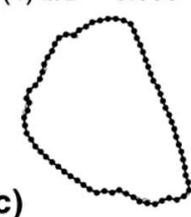

(2) $b / D=0.500$

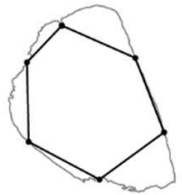

(5) $b / D=0.010$

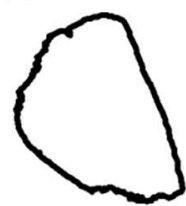

(3) $b / D=0.100$

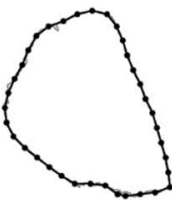

(6) $b / D=0.001$

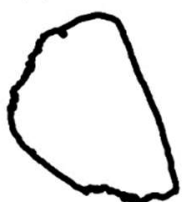

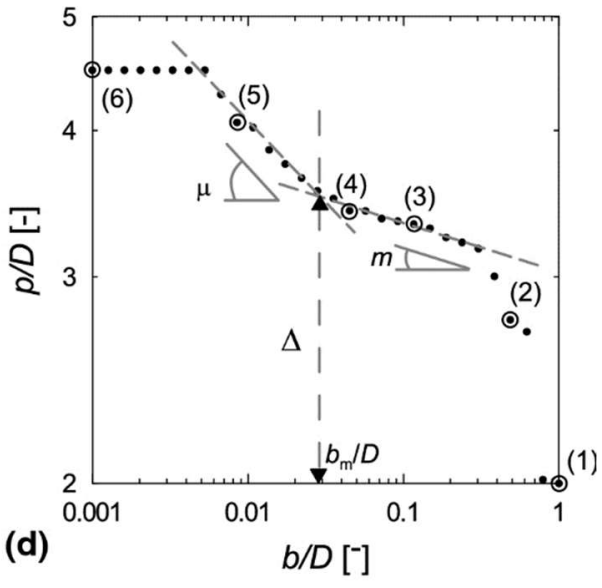

Figure 2. Toyoura sand particle: (a) SEM image; (b) segmented image; (c) perimeter at different stick lengths; c) $\log (p / D)$ vs $\log (b / D)$.

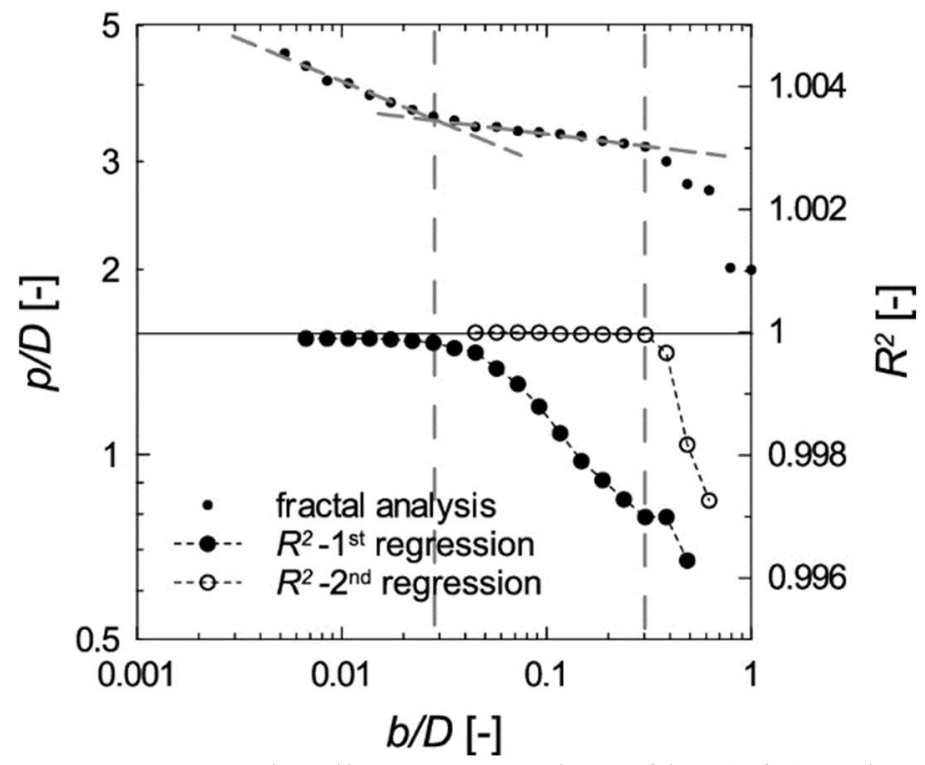

Figure 3. Moving linear regression of $\log (p / D)$ vs $\log (b / D)$ data. 

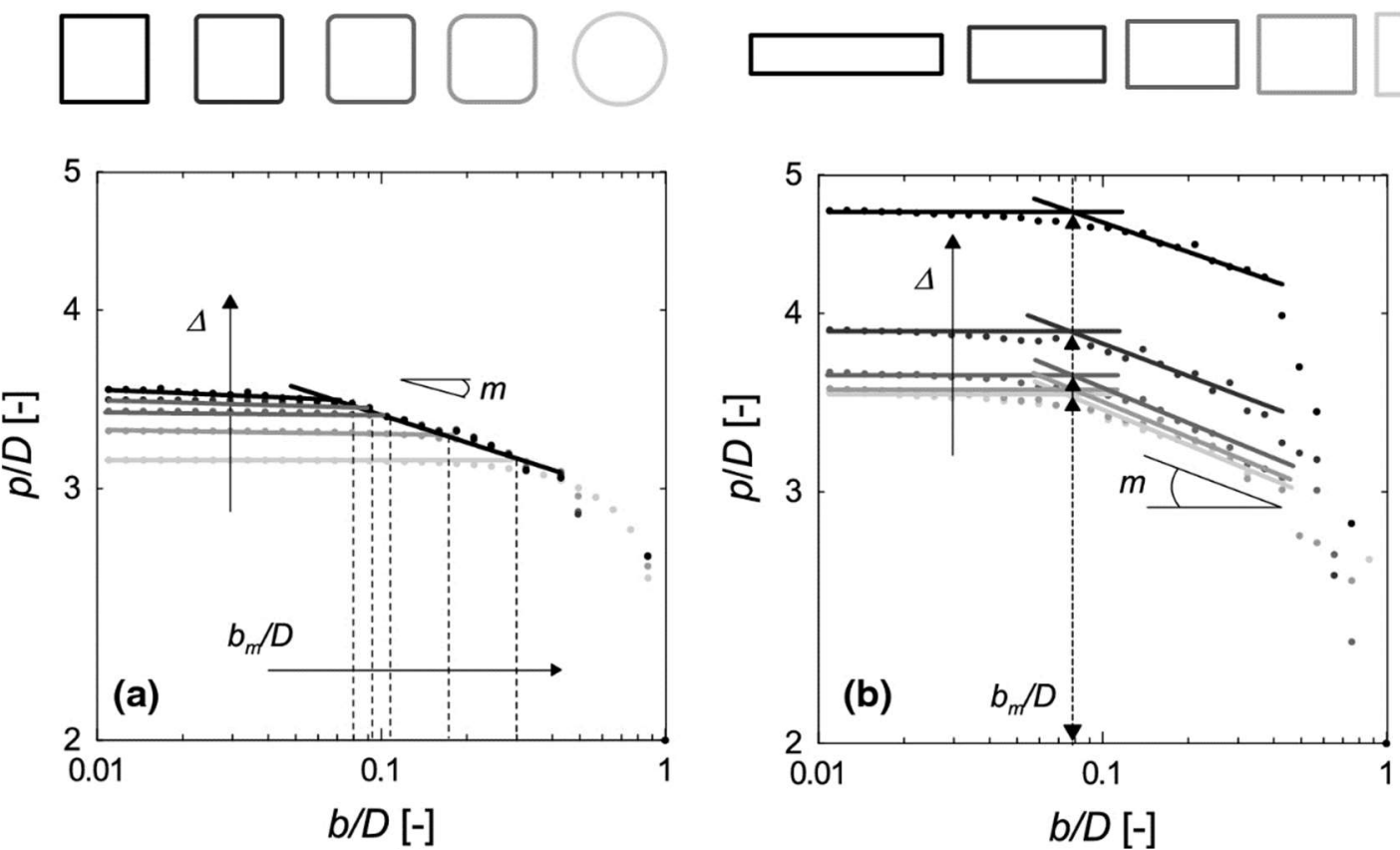

Figure 4. Smooth shapes: (a) family of squares with prograssively rounded corners; (b) family of rectangles of increasing elongation.

(a)

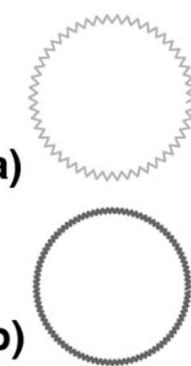

(c)
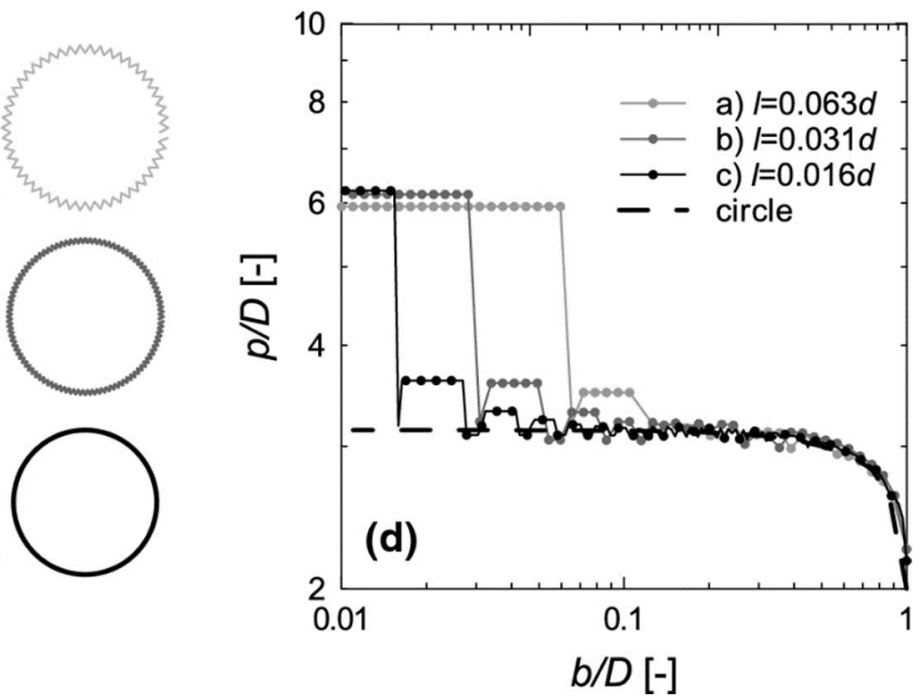

Figure 5. Circles with saw tooth roughness of decreasing size. 


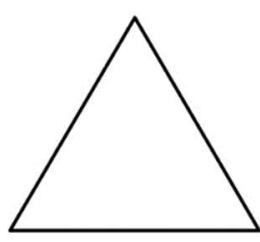

(a)

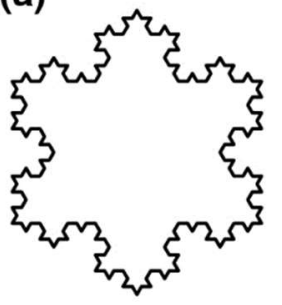

(d)

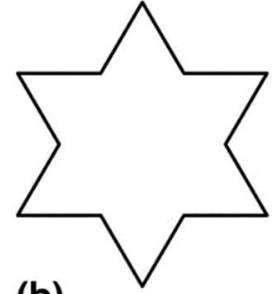

(b)

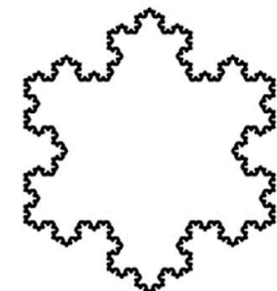

(e)

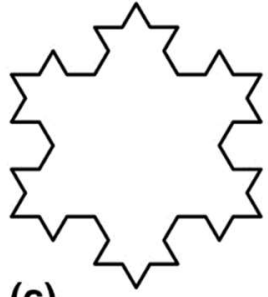

(c)

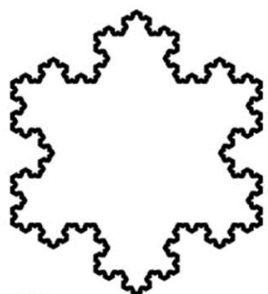

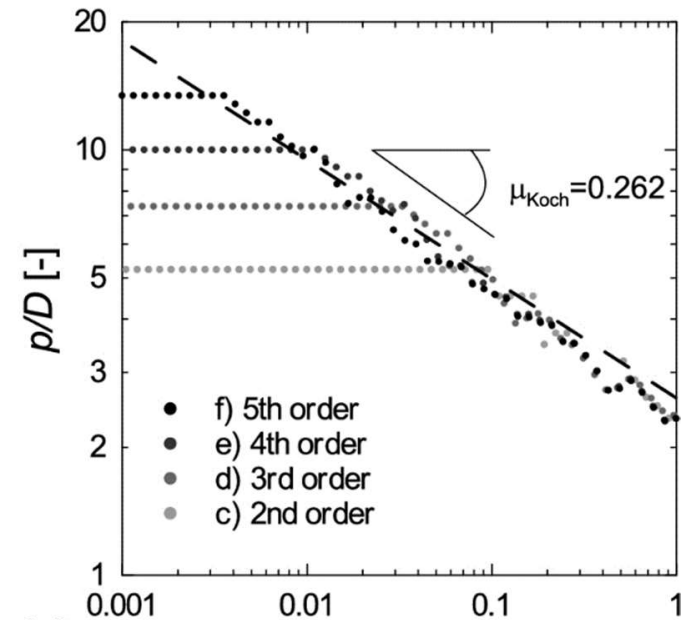

(g)

$b / D[-]$

Figure 6. (a) to (f) Euclidean approximations of Koch snow flake at increasing order, and (g) fractal analysis of their contour.

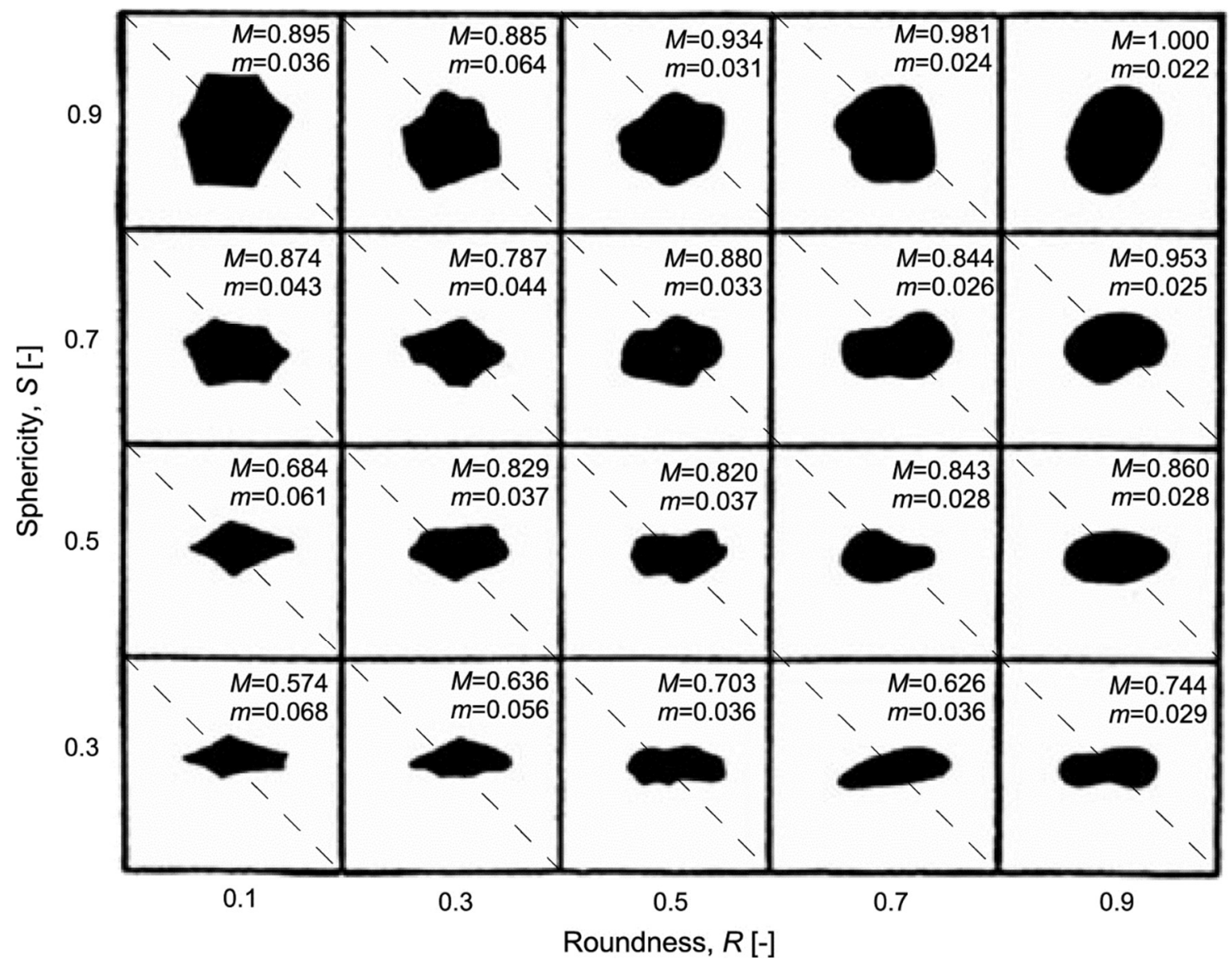

Figure 7. $M$ and $m$ for Krumbein \& Sloss chart (1936) particles. 

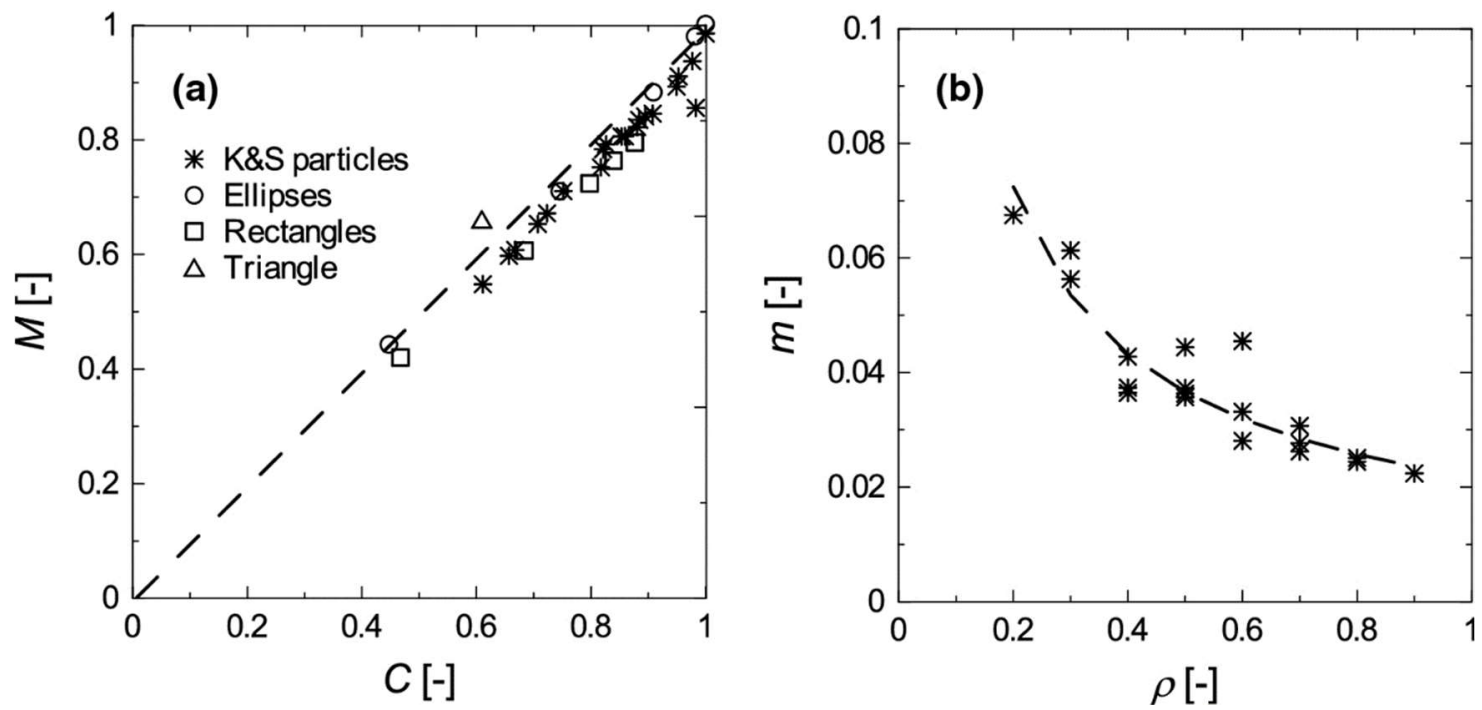

Figure 8. Relationships between: (a) descriptor $M$ and circularity $C$, (b) descriptor $m$ and regularity $\rho$.
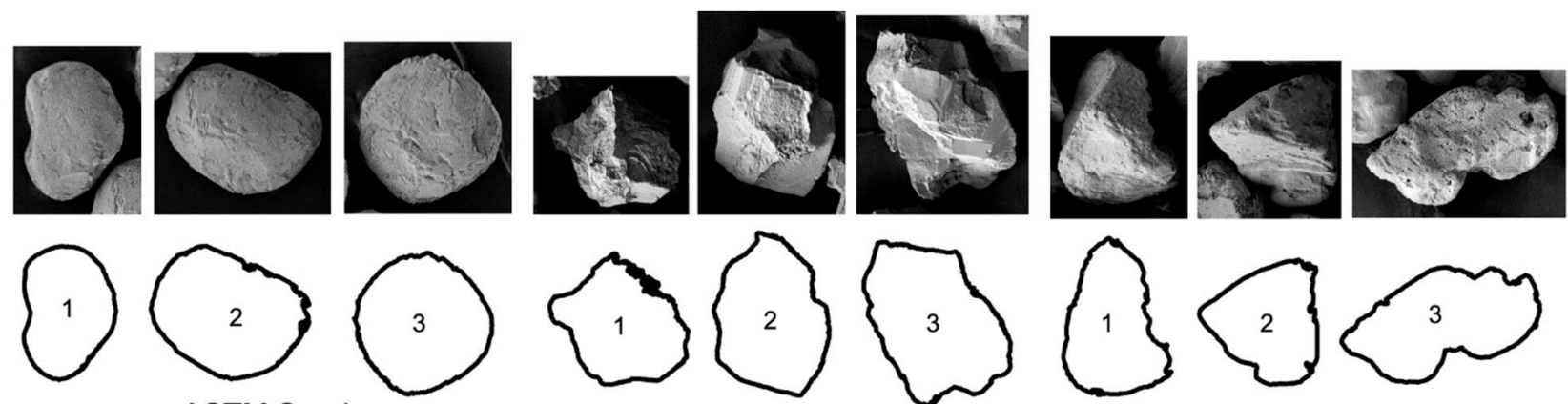

ASTM Sand
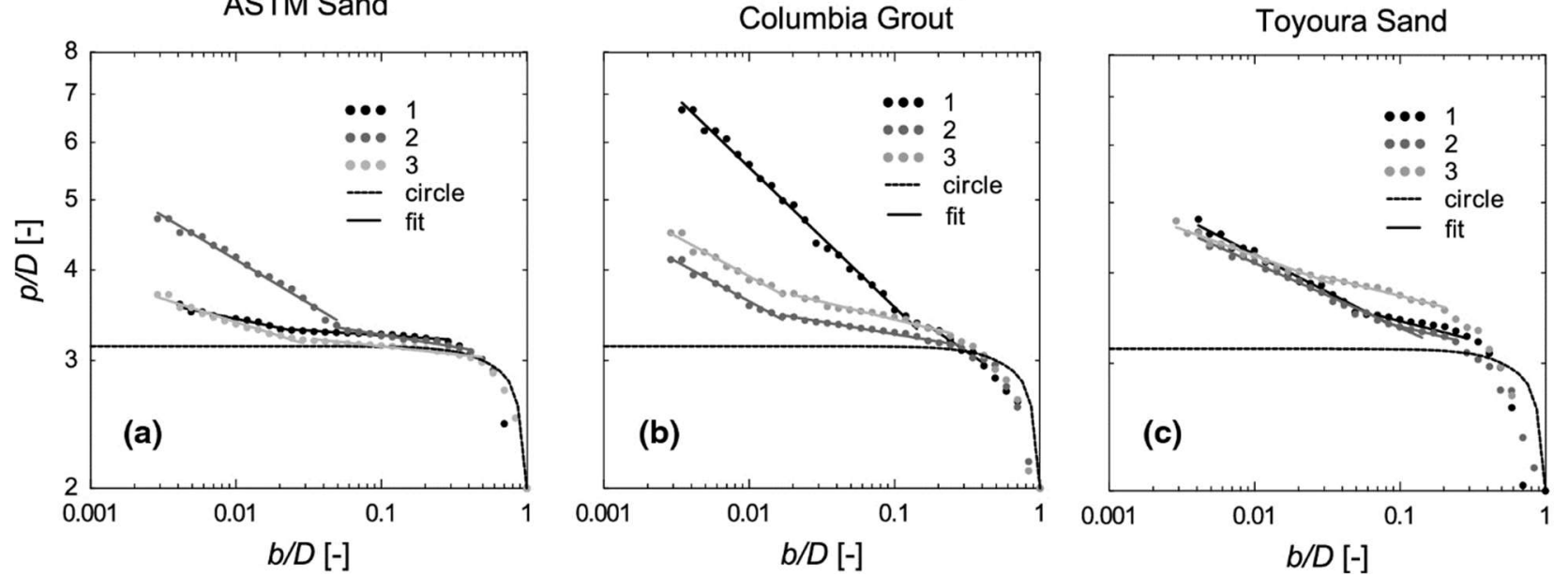

Figure 9. Natural sand particles: (a) ATSM Sand, (b) Columbia Grout, and (c) Toyoura Sand. 

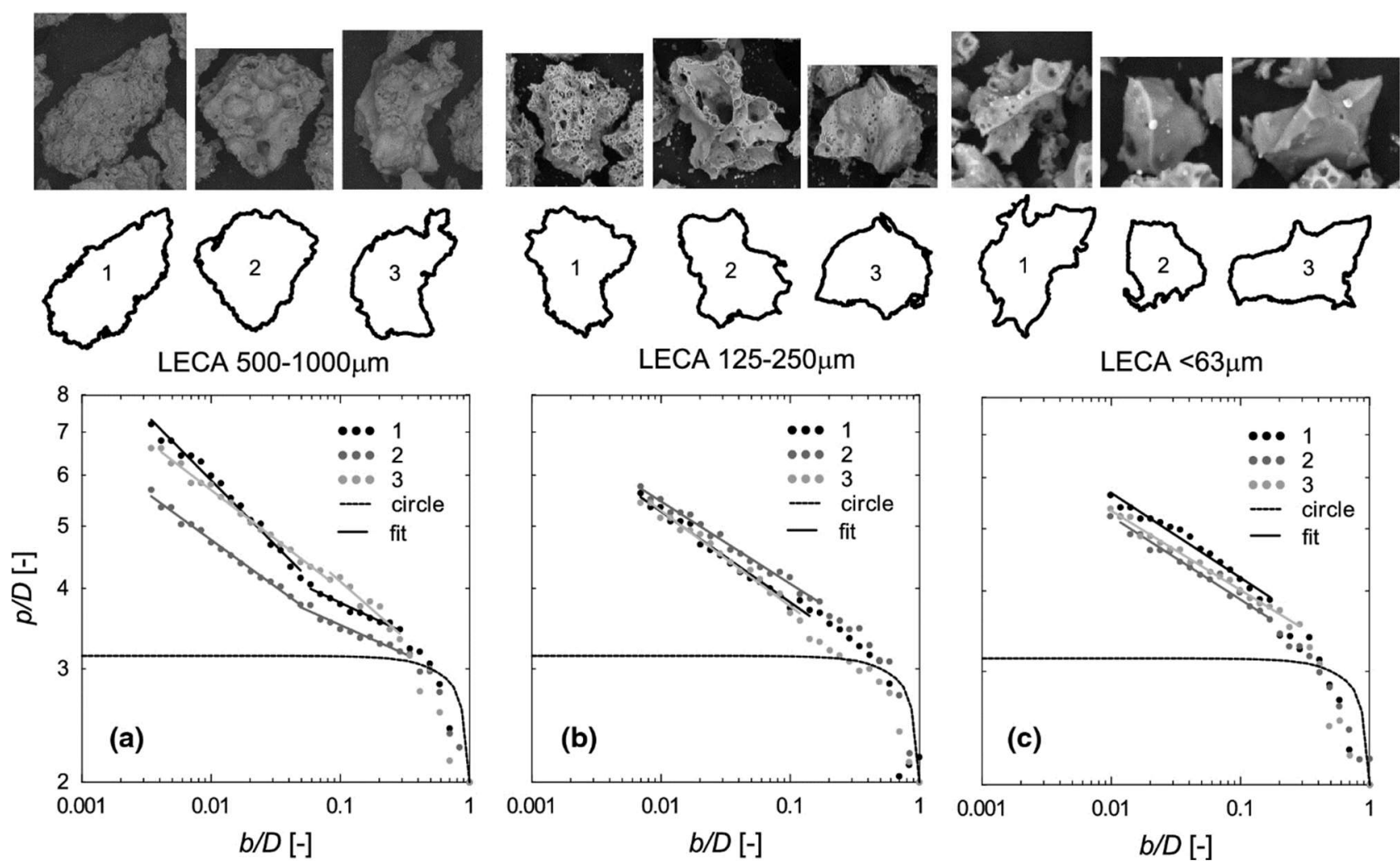

Figure 10. Crushed LECA in different grain sizes: (a) 500-1000 $\mu \mathrm{m}$, (b) $125-250 \mu \mathrm{m}$, and (c) 63 $\mu \mathrm{m}$.

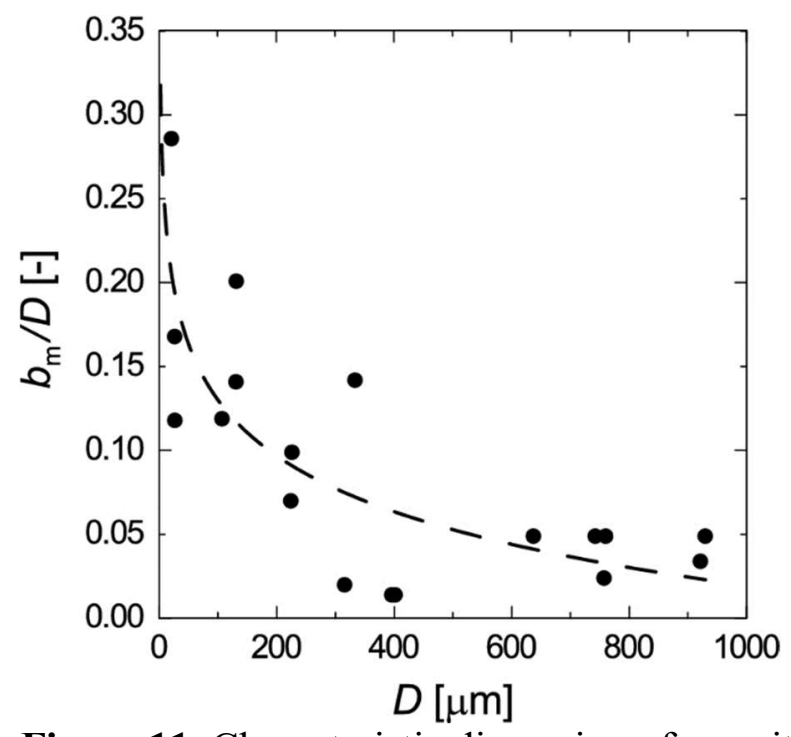

Figure 11. Characteristic dimension of asperities $b_{m} / D$ as a function of particle dimension $D$. 